\title{
Insight into Lithium Transport in Lithium Nitridometallate Battery Materials from Muon Spin Relaxation
}

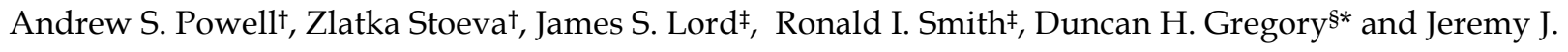
Titman $^{* *}$

tSchool of Chemistry, University of Nottingham, University Park, Nottingham, NG7 2RD, UK

‡ISIS Facility, Rutherford Appleton Laboratory, Chilton, Oxfordshire OX11 0QX, UK

sWESTChem, School of Chemistry, University of Glasgow, Glasgow, G12 8QQ, UK

\begin{abstract}
Muon spin relaxation and powder neutron diffraction have been combined to study three lithium cobalt nitride battery materials. Neutron diffraction shows that these retain the $P 6 / m m m$ space group of $\mathrm{Li}_{3} \mathrm{~N}$ with Co located only on $\operatorname{Li}(1)$ sites. The lattice parameters vary smoothly with the degree of metal substitution, such that the $\left[\mathrm{Li}_{2} \mathrm{~N}\right]$ layers expand while the layer separation contracts, as observed previously for similar series of $\mathrm{Cu}$ - and $\mathrm{Ni}$-substituted materials. However, in contrast to the latter, the $\mathrm{Li}_{3-\mathrm{x}-\mathrm{y}} \mathrm{Co}_{\mathrm{x}} \mathrm{N}$ phases exhibit Curie-Weiss paramagnetism and this prevents the use of nuclear magnetic resonance to measure $\mathrm{Li}^{+}$ transport parameters. Therefore, muon spin relaxation has been employed here as an alternative technique to obtain quantitative information about $\mathrm{Li}^{+}$diffusion. Muon spin relaxation shows that $\mathrm{Li}^{+}$diffusion in $\mathrm{Li}_{3-\mathrm{x}-}$ ${ }_{y} \mathrm{Co}_{x} \mathrm{~N}$ is anisotropic with transport confined to the $\left[\mathrm{Li}_{2} \mathrm{~N}\right]$ plane at low temperature and exchange between $\operatorname{Li}(1)$ and $\operatorname{Li}(2)$ sites dominant at high temperature. By a comparison with previous studies some general trends have been established across a range of $\mathrm{Cu}-$, Ni- and Co-substituted materials. For intra-layer diffusion $\mathrm{E}_{\mathrm{a}}$ decreases as metal substitution increases and the corresponding expansion of the layers results in a more open pathway for $\mathrm{Li}^{+}$diffusion. However, an optimal value of $\mathrm{x}$ is found with $a \approx 3.69 \AA$ after which the concomitant contraction in layer spacing reduces the polarizability of the lattice framework.
\end{abstract}




\section{Introduction}

The materials used to manufacture electrodes for lithium batteries usually contain paramagnetic transition metal centres which provide the redox mechanism associated with lithium insertion and extraction. Unfortunately, paramagnetism generally precludes the measurement of $\mathrm{Li}^{+}$ion transport parameters by ${ }^{7} \mathrm{Li}$ nuclear magnetic resonance (NMR) because interactions with the metal electronic moments mask the effects of ion dynamics on spin relaxation rates and spectral linewidths. ${ }^{1}$ However, muon spin relaxation ( $\mu$ SR) has been suggested recently as an alternative means of studying $\mathrm{Li}^{+}$transport in these circumstances..$^{2-4} \mathrm{In} \mu \mathrm{SR}$ experiments $100 \%$ spin polarized muons are implanted into materials where they thermalize, allowing the measurement of local magnetic fields. The radioactive decay of the muons results in the emission of positrons along the spin polarization direction which are detected by a set of scintillators arranged around the sample. Hence, the histogram of the muon decay asymmetry measured in this way gives the evolution of the muon spin polarization. The spin polarization precesses in a transverse magnetic field (TF experiment geometry), and the precession spectrum is determined by the applied field and the local fields due to magnetic moments in the sample. In zero field (ZF geometry), on average only two-thirds of local fields in a powdered sample are perpendicular to the initial muon spin polarization. For a Gaussian distribution of local fields, this situation is described by the Kubo-Toyabe depolarization function. ${ }^{5}$ A longitudinal magnetic field can be applied parallel to the muon spin polarization (LF geometry) with sufficient strength to quench the interaction of the muon with local fields due to nuclear moments. This allows the electronic contribution to the muon spin relaxation to be separated for paramagnetic materials, so that $\mathrm{Li}^{+}$ion transport can be studied. In the first application of $\mu \mathrm{SR}$ to battery materials Kaiser et al. ${ }^{2}$ detected the onset of $\mathrm{Li}^{+}$mobility in the "charged" $(x=0.2)$ and "empty" $(x=1.0)$ states of lithium manganese oxide $\mathrm{Li}_{x}\left[\mathrm{Li}_{0.04} \mathrm{Mn}_{1.96}\right] \mathrm{O}_{4}$ for which $\mathrm{Li}$ substitution onto the $\mathrm{Mn}$ site results in improved cycling performance. More recently, both Sugiyama et $a l .{ }^{3}$ and Powell et al. ${ }^{4}$ have used $\mu$ SR for the first time to quantify $\mathrm{Li}^{+}$diffusion coefficients and activation energies, the former in lithium cobalt oxide $\mathrm{Li}_{x} \mathrm{CoO}_{2}$ with $\mathrm{x}=0.53$ and 0.73 and the latter in Ni-substituted lithium nitride materials $\mathrm{Li}_{3-\mathrm{x}-\mathrm{y}} \mathrm{Ni}_{\mathrm{x}} \mathrm{N}$ with $\mathrm{x}=0.36$ and 0.57 .

In this paper we report a combined powder neutron diffraction (PND) and zero-field (ZF) $\mu S R$ study on three paramagnetic lithium cobalt nitride materials with $x$ between 0.2 and $0.6 . \mathrm{Li}_{2.6} \mathrm{Co}_{0.4} \mathrm{~N}$ has been suggested as an improved anode material, 6,7 in particular because of its high capacity which can exceed 700 $\mathrm{mA} \mathrm{h} \mathrm{g}^{-1}$. More recently, single crystals and bulk powders of similar ternary lithium nitridometallate phases 
$\mathrm{Li}_{3-\mathrm{x}-\mathrm{y}} \mathrm{M}_{\mathrm{x}} \mathrm{N}$ (where $\mathrm{M}=\mathrm{Cu}, \mathrm{Ni}$ or $\mathrm{Co}$ and $\mathrm{y}$ represents a lithium vacancy) have been synthesized with controlled and reproducible vacancy and substitution levels. ${ }^{8,9}$ X-ray and neutron diffraction studies ${ }^{10}$ of these materials indicate defect structures which retain the $P 6 / \mathrm{mmm}$ space group of $\mathrm{Li}_{3} \mathrm{~N}^{11}$ (Figure 1) with $\mathrm{M}$ located only on $\mathrm{Li}(1)$ sites and disordered $\mathrm{Li}(2)$ vacancies in the $\left[\mathrm{Li}_{2} \mathrm{~N}\right]$ plane. The vacancy concentrations are significantly higher than in $\mathrm{Li}_{3} \mathrm{~N}$ with implications for the redox chemistry and $\mathrm{Li}^{+}$conductivity which determine their use as anode materials.

Variable temperature measurements of the ${ }^{7} \mathrm{Li} \mathrm{NMR}$ linewidth have illuminated some aspects of the $\mathrm{Li}^{+}$ diffusion dynamics, particularly for diamagnetic Cu-substituted phases. ${ }^{12}$ However, for paramagnetic Li3-x${ }_{y} \mathrm{Co}_{x} \mathrm{~N}$, the ${ }^{7} \mathrm{Li}$ NMR linewidth varies with temperature (Figure S1 Supplementary Information) according to a Curie-Weiss law, indicating that any effect of dynamics is obscured by interactions with the Co electronic moments. As described above this problem can be completely circumvented by the use of $\mu \mathrm{SR}$, since the electronic contribution to the muon spin relaxation can be measured independently via complementary LF experiments. This allows the effects of $\mathrm{Li}^{+}$dynamics on the muon depolarization to be resolved, and otherwise unobtainable diffusion coefficients and activation energies to be measured. In this paper we describe a combined $\mu \mathrm{SR}$ and neutron diffraction study of two $\mathrm{Li}_{3-x-y} \mathrm{Co}_{x} \mathrm{~N}$ phases with the aim of understanding the links between their structure, stoichiometry and transport properties. In addition, we compare diffusion coefficients and activation energies measured by both $\mu \mathrm{SR}$ and NMR for a series of nitridometallate $\mathrm{Li}_{3-x-y} \mathrm{M}_{x} \mathrm{~N}(\mathrm{M}=\mathrm{Cu}, \mathrm{Ni}, \mathrm{Co})$ materials previously characterized by neutron diffraction. This allows us to draw some general conclusions about trends in these parameters with metal substitution and vacancy concentration.

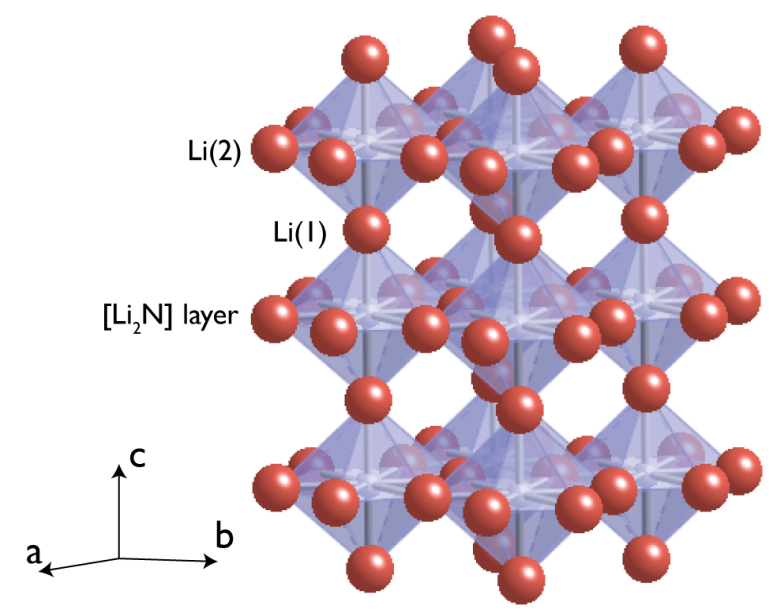


Figure 1. (Colour) Crystal structure of $\alpha-\mathrm{Li}_{3} \mathrm{~N} .^{2}$ The (blue) polyhedra illustrate the hexagonal bipyramidal co-ordination of the nitrogen atoms (small blue spheres) by lithium atoms (large red spheres) in the $\mathrm{P} 6 / \mathrm{mmm}$ structure. Note the $\left[\mathrm{Li}_{2} \mathrm{~N}\right]$ layers perpendicular to the crystallographic $c$ axis. Intra-layer Li sites are designated $\mathrm{Li}(2)$, inter-layer ones $\mathrm{Li}(1)$.

\section{Experimental}

Synthesis. $\mathrm{Li}_{3} \mathrm{~N}$ was prepared as described elsewhere ${ }^{13}$ by reaction of molten lithium-sodium alloy with dry nitrogen at $720 \mathrm{~K}$ for 4 days. Subsequently, excess sodium was removed by vacuum distillation at $670-720$ $\mathrm{K}$ for $24 \mathrm{hr}$. The product was identified as single-phase $\alpha-\mathrm{Li}_{3} \mathrm{~N}$ using powder $\mathrm{X}$-ray diffraction. $\mathrm{Li}_{3-\mathrm{x}-\mathrm{y}} \mathrm{Co}_{\mathbf{x}} \mathrm{N}$ phases with nominal $\mathrm{x}=0.2,0.4$ and 0.6 (denoted $\mathbf{1}-\mathbf{3}$, respectively) were synthesized by heating a stoichiometric mixture of $\mathrm{Li}_{3} \mathrm{~N}$ and cobalt powder (Goodfellows, 99.8\%, $1.6 \mu \mathrm{m}$ particle size). These were ground and pressed into a pellet which was loaded into an alumina crucible, sealed in a stainless steel reaction vessel and heated at $960 \mathrm{~K}$ for 6 days. Syntheses generally required repeated regrinding of the reaction mixture. All synthetic work and sample preparation was carried out in a nitrogen-filled glove box (Saffron Scientific recirculating glovebox; $\mathrm{O}_{2}<1$ ppm, $\mathrm{H}_{2} \mathrm{O}<5$ ppm).

X-ray and Neutron Diffraction. Powder X-ray diffraction (PXD) data for $\mathbf{1}$ - $\mathbf{3}$ were collected at ambient temperature on a Philips XPERT $\theta$ - $2 \theta$ diffractometer using $\mathrm{Cu}$ K $\alpha$ radiation. The air- and moisture-sensitive products were sealed in custom-designed sample holders during data collection. ${ }^{14}$ Phase purity was evaluated by taking $2 \mathrm{hr}$ scans over the range $5^{\circ}-100^{\circ} 2 \theta$. These were cross-referenced to the ICDD PDF database via the Philips PC-IDENTIFY routine and to powder patterns generated from the Inorganic Crystal Structure Database (ICSD) using PowderCell 3.15 Scan times of 12 hr were used to obtain data suitable for Rietveld refinements which were carried out using the General Structure Analysis System (GSAS) ${ }^{16}$ via the EXPGUI interface. ${ }^{17}$ Initial structural models were based on $\alpha-\mathrm{Li}_{3} \mathrm{~N}$ with the inter-layer $\operatorname{Li}(1)$ site partially substituted by Co and fractional occupancies estimated from the nominal compositions. Lattice parameters for model structures were obtained by indexing the powder patterns using DICVOL91 ${ }^{18}$.

Time-of-flight PND data for $\mathbf{1}$ - 3 were collected at $250 \mathrm{~K}$ using the high-intensity diffractometer POLARIS at the ISIS neutron facility in the Rutherford Appleton Laboratory. Powder samples ca. 1.5 - 2 g each were sealed into thin-walled vanadium cans with indium gaskets. Data were collected using three fixed detector banks at $<2 \theta>=35^{\circ}, 90^{\circ}$ and $145^{\circ}$. Rietveld refinements were performed using the structures obtained from PXD as a starting point, according to procedures outlined for Ni-substituted $\mathrm{Li}_{3} \mathrm{~N}$ phases in Ref. ${ }^{13}$ Data from 
all three detector banks was refined simultaneously. Note that the vanadium sample can yielded a group of reflections that were excluded from the refinements, but the excluded regions did not contain reflections arising from the compounds under study.

Magnetic Susceptibility. Magnetic susceptibility measurements were performed on a Quantum Design MPMS XL 5T SQUID magnetometer in the temperature range $2-250 \mathrm{~K}$ at an applied magnetic field of $100 \mathrm{mT}$. The samples were loaded into airtight gelatine capsules. The data were corrected for the diamagnetic contributions from the gelatine containers.

Muon Spin Relaxation. The MUSR instrument at the ISIS pulsed muon facility in the Rutherford Appleton Laboratory was used to make $\mu S R$ measurements. On the MUSR instrument there are 64 positron scintillation detectors in two circular arrays, and data acquisition occurs via 64 TDCs, recording about $2.5 \mathrm{x}$ $10^{7}$ events per hour. Helmholtz coils are used to apply magnetic fields up to $250 \mathrm{mT}$ in transverse or longitudinal experiment geometry and to compensate for the earth's magnetic field in zero field. A closed cycle helium refrigerator was used to regulate the sample temperature between $15 \mathrm{~K}$ and $340 \mathrm{~K}$, while a furnace was used to obtain higher temperatures. The $\mu \mathrm{SR}$ experiments were carried out on samples 2 and 3 at temperatures between $20 \mathrm{~K}$ and $540 \mathrm{~K}$. Approximately $2 \mathrm{~g}$ of each was packed in a nitrogen-filled glove box into airtight titanium sample holders with titanium foil windows. Transverse field (TF) measurements used a field of $2.4 \mathrm{mT}$ and typically $5 \times 10^{6}$ events were recorded for each temperature studied. These were used to calibrate a correction factor $\alpha$ which accounts for the relative efficiency of the forwards and backwards groups of scintillation detectors. In contrast zero field (ZF) experiments required at least $1 \times 10^{7}$ events for sufficient signal. Longitudinal field (LF) measurements with fields up to $100 \mathrm{mT}$ were also recorded at each temperature and used to quantify the paramagnetic contribution to muon relaxation. Data analysis was carried out using the WiMDA programme. ${ }^{19}$

\section{Results and Discussion}

Crystal Structure. PXD data for 1 - 3 yielded powder patterns (Figure S2 Supplementary Information) showing single-phase samples which were successfully indexed in the $\alpha-\mathrm{Li}_{3} \mathrm{~N}$ space group $P 6 / \mathrm{mmm}$. No reflections for Co metal were observed for samples with $x \leq 0.7$ which is the highest substitution level reported to date for the $\mathrm{Li}_{3-x-y} \mathrm{Co}_{x} \mathrm{~N}$ series. Critical factors for obtaining high $\mathrm{x}$ were found to be the use of $\mathrm{Co}$ metal powders with particle sizes $\leq 2 \mu \mathrm{m}$ and high reaction temperature (950 - $970 \mathrm{~K})$. Use of larger particle sizes limited the substitution level to $x \approx 0.5$ in line with previous reports, ${ }^{20,21}$ while reaction temperatures 
below $920 \mathrm{~K}$ produced samples with Co metal impurities. This is in contrast to the $\mathrm{Li}_{3-x-y} \mathrm{Ni}_{x} \mathrm{~N}$ materials which are easily obtained as single phases across a much wider temperature range, from $820 \mathrm{~K}$ to $990 \mathrm{~K} .13,22$

While PXD gave an initial model, fine tuning the structure requires neutron diffraction in order to precisely locate the light $\mathrm{Li}$ atoms, to accurately quantify vacancy concentrations and to obtain improved thermal displacement parameters. PND refinements using lattice parameters from PXD confirmed the retention of the $\mathrm{Li}_{3} \mathrm{~N}$ structure with Co occupying the inter-layer $\operatorname{Li}(1)$ site only. Profile plots for Rietveld refinement against PND data for a representative sample $\left(\mathrm{Li}_{3-x-y} \mathrm{Co}_{x} \mathrm{~N}\right.$ with $\left.\mathrm{x}=0.58,3\right)$ are shown in the Supplementary Information (Figure S3). The resulting crystallographic parameters at $250 \mathrm{~K}$ are given in Table 1, and calculated inter-atomic distances and bond angles are in Table 2. Refined atomic parameters are in the Supplementary Information (Table S1). The lattice parameters vary smoothly with $\mathrm{x}$ in common with $\mathrm{Cu}-$ and Ni-substituted materials, as shown in Figure 2 where results from this work have been combined with those of our previous PND studies.10,12,13 In all cases the $a$ parameter, which depends on the $\operatorname{Li}(2)-\mathrm{N}$ distance, increases with the degree of substitution, while the $c$ parameter, which corresponds to the interlayer separation, contracts.

\begin{tabular}{|c|c|c|c|}
\hline Instrument, radiation & POLARIS T & F diffactom & er, neutron \\
\hline Sample, nominal composition, & $1, x=0.2$ & $2, x=0.4$ & $3, x=0.6$ \\
\hline $\mathrm{T} / \mathrm{K}$ & \multicolumn{3}{|c|}{250} \\
\hline Crystal System & \multicolumn{3}{|c|}{ Hexagonal } \\
\hline Space Group & \multicolumn{3}{|c|}{$P 6 / \mathrm{mmm}$} \\
\hline $\mathrm{Z}$ & \multicolumn{3}{|c|}{1} \\
\hline Co substitution level (x) & $0.202(3)$ & $0.428(2)$ & $0.581(2)$ \\
\hline $\mathrm{Li}^{+}$vacancy concentration $(\mathrm{y})$ & $0.040(7)$ & $0.240(6)$ & $0.436(2)$ \\
\hline $\mathrm{M} / \mathrm{g} \mathrm{mol}^{-1}$ & 45.049 & 55.408 & 61.983 \\
\hline a lattice parameter/ $\AA$ & $3.68852(4)$ & $3.71281(4)$ & $3.73663(3)$ \\
\hline c lattice parameter $/ \AA$ & $3.79377(7)$ & $3.71888(8)$ & $3.64012(5)$ \\
\hline Unit cell volume V / $\AA^{3}$ & $44.700(1)$ & $44.396(1)$ & $44.016(1)$ \\
\hline Number of observations & 14299 & 13298 & 13644 \\
\hline $\mathrm{R}_{\mathrm{wp}}$ & 0.0087 & 0.0104 & 0.0138 \\
\hline$\chi^{2}$ & 1.107 & 1.113 & 1.585 \\
\hline
\end{tabular}

Table 1. Crystallographic parameters obtained from Rietveld refinements against powder diffraction data for $\mathrm{Li}_{3-\mathrm{x}-\mathrm{y}} \mathrm{Co}_{x} \mathrm{~N}$ materials $\mathbf{1}-3$.

\begin{tabular}{|l|c|c|c||}
\hline \hline Sample, composition & $\mathbf{1 , x}=0.20$ & $\mathbf{2 ,} \mathrm{x}=0.43$ & $\mathbf{3 , x}=0.58$ \\
\hline \hline$(\mathrm{Li}, \mathrm{Co})(1)-\mathrm{N} \times 2 / \AA$ & $1.8969(1)$ & $1.8594(1)$ & $1.8201(1)$ \\
\hline $\mathrm{Li}(2)-\mathrm{N} \times 3, / \AA$ & $2.1295(2)$ & $2.1434(1)$ & $2.1574(2)$ \\
\hline $\mathrm{Li}(2)-\mathrm{Li}(2) \times 3 / \AA$ & $2.1295(2)$ & $2.1434(1)$ & $2.1574(2)$ \\
\hline$(\mathrm{Li}, \mathrm{Co})(1)-\mathrm{Li}(2) \times 6 / \AA$ & $2.8518(2)$ & $2.8375(1)$ & $2.8225(2)$ \\
\hline$(\mathrm{Li}, \mathrm{Co})(1)-\mathrm{N}-(\mathrm{Li}, \mathrm{Co})(1) /{ }^{\circ}$ & 180 & 180 & 180 \\
\hline $\mathrm{Li}(2)-\mathrm{N}-\mathrm{Li}(2) /^{\circ}$ & 120 & 120 & 120 \\
\hline
\end{tabular}


Table 2. Selected interatomic distances and bond angles from Rietveld refinements for $\mathrm{Li}_{3-\mathrm{x}-\mathrm{y}} \mathrm{Co}_{\mathrm{x}} \mathrm{N}$ materials $\mathbf{1}$ $-3$.

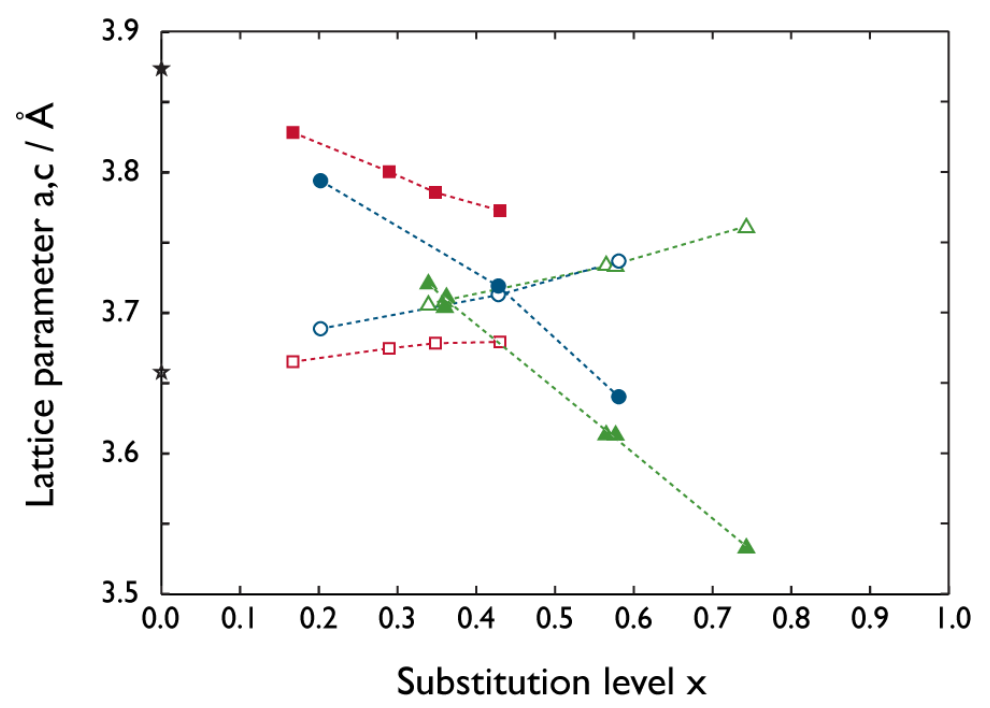

Figure 2. (Colour) Lattice parameters a (open symbols) and $c$ (filled symbols) measured by neutron diffraction for $\mathrm{Li}_{3-\mathrm{x}-\mathrm{y}} \mathrm{M}_{\mathrm{x}} \mathrm{N}$ as a function of $\mathrm{x}$ for $\mathrm{M}=\mathrm{Co}$ (blue circles), $\mathrm{Ni}$ (green triangles) and $\mathrm{Cu}$ (red squares), as well as for $\mathrm{Li}_{3} \mathrm{~N}$ (black stars). Lines are drawn to guide the eye. Additional data was taken from Refs. ${ }^{10}, 12$ and ${ }^{13}$.

Magnetic Susceptibility. The temperature variation for the magnetic susceptibility of all the $\mathrm{Li}_{3-\mathrm{x}-\mathrm{y}} \mathrm{Co}_{\mathrm{x}} \mathrm{N}$ materials 1 - 3 (see Supplementary Information Figure S4) follows a modified Curie-Weiss law $\chi=\chi_{0}+\mathrm{C} /(\mathrm{T}$ - $\theta$ ) where $\chi_{0}$ is a temperature-independent contribution, and C and $\theta$ are the Curie and Weiss constants, respectively. Values for these, along with the effective magnetic moment $\mu_{\text {eff }}$ per Co atom, are given in Table 3. Both $\mathrm{C}$ and $\mu_{\text {eff }}$ depend strongly on composition, and the large values at low $\mathrm{x}$ are not consistent with the existence of exclusively $\operatorname{Co}(\mathrm{I})$ species with spin-only contributions $\left(\mathrm{S}=1, \mu_{\mathrm{eff}}=2.83 \mu_{\mathrm{B}}\right)$. Although an orbital contribution to the moment has been suggested, ${ }^{23}$ the presence of higher oxidation states Co(II) or Co(III) is also possible, and this picture is supported by the significant vacancy concentration at the Li(2) site evident in the refined structural data. Note that electron energy loss spectroscopy studies of $\mathrm{Li}_{2.6-\mathrm{y}} \mathrm{Co}_{0.4} \mathrm{~N}$ also reveal multiple spin states for Co. ${ }^{24}$ The magnetic properties of the $\mathrm{Li}_{3-\mathrm{x}-\mathrm{y}} \mathrm{Co}_{\mathrm{x}} \mathrm{N}$ series contrast with those reported previously $^{13}$ for the corresponding Ni-substituted materials which show a temperature-independent $\chi$ with only a small Curie-Weiss contribution below $20 \mathrm{~K}$. This indicates a change from Pauli paramagnetism with substantial electron delocalization to Curie-Weiss behaviour with more isolated transition metal centres on replacement of $\mathrm{Ni}$ by $\mathrm{Co}$. For both series, however, $\mu_{\mathrm{eff}}$ decreases as $\mathrm{x}$ increases, becoming smaller than the 
relevant spin-only value at $\mathrm{x} \approx 0.5$ for $\mathrm{Co}$, suggesting progressive delocalization as the substitution level increases.

\begin{tabular}{|c|c|c|c|}
\hline Sample, composition & $1, x=0.20$ & $2, x=0.43$ & $3, x=0.58$ \\
\hline$\chi_{0} /$ emu $\mathrm{mol}^{-1}$ & $0.0026(2)$ & $-0.0015(3)$ & $-0.0008(2)$ \\
\hline $\mathrm{C} / \mathrm{emu} \mathrm{mol}-1 \mathrm{~K}$ & $2.47(9)$ & $1.60(3)$ & $0.93(1)$ \\
\hline$\theta / \mathrm{K}$ & $-2.4(1)$ & $-2.8(3)$ & $-1.3(2)$ \\
\hline$\mu_{\text {eff }}$ per Co atom / $\mu_{\mathrm{B}}$ & 4.45 & 3.58 & 2.73 \\
\hline
\end{tabular}

Table 3. Magnetic susceptibilitya of $\mathrm{Li}_{3-x-y} \mathrm{Co}_{x} \mathrm{~N}$ materials 1 - 3.

a. The temperature variation of $\chi$ is described in terms of a modified Curie-Weiss law $\chi=\chi_{0}+C /(T-\theta)$ where the terms are defined in the text.

TF $\mu$ SR Experiments. All TF $\mu$ SR signals gave an initial positron count asymmetry of approximately $25 \%$ and showed a Larmor oscillation at a frequency of $0.33 \mathrm{MHz}$. This indicates that the muons implant into both samples (2 and 3$)$ as diamagnetic $\mu^{+}$across the whole of the temperature range studied. In addition to a Gaussian envelope due to the local nuclear field distribution, the TF $\mu$ SR signals decayed exponentially as a result of the fluctuating Co electronic moments. This behaviour has been observed previously for lithium manganate spinels. ${ }^{2}$

ZF and LF $\mu S R$ Experiments. Figure 3 shows $\mu S R$ signals for the $\mathrm{Li}_{3-x-y} \mathrm{Co}_{x} \mathrm{~N}$ material with $\mathrm{x}=0.58$ (3) recorded at $20 \mathrm{~K}$ in ZF (open circles) and $100 \mathrm{G}$ LF (closed circles). As with TF experiments the initial positron asymmetry was approximately $25 \%$ across the whole temperature range, confirming that muons implant as diamagnetic $\mu^{+}$. The total relaxing fraction measured from the ZF $\mu$ SR signals was only about $50 \%$, since a proportion of the muons are stopped in the sample holder where they do not relax. The static Kubo-Toyabe function $^{5}$ is given by

$\mathrm{G}_{\mathrm{z}}(\Delta, \mathrm{t})=\frac{1}{3}+\frac{2}{3}\left(1-\gamma_{\mu}^{2} \Delta^{2} \mathrm{t}^{2}\right) \exp \left(-\frac{1}{2} \gamma_{\mu}^{2} \Delta^{2} \mathrm{t}^{2}\right)$

where $\Delta$ is the depolarization rate which is related to the second moment of the Gaussian local field distribution. However, the ZF signal in Figure 3 (open circles) never fully recovers to the value of $1 / 3$ predicted by Eq. (1), so the depolarization function was modified to take account of muon dynamics which can be included by assuming a Markovian strong collision model. ${ }^{5}$ The dynamic Kubo-Toyabe function which depends on the muon hopping rate $v$ cannot be obtained in closed form and must be generated during fitting by a numerical inverse Laplace transform. ${ }^{19}$ The dynamic Kubo-Toyabe behaviour observed 
in ZF (open circles) is damped by an exponential decay in muon polarization which, as in the TF case, arises from fluctuating Co electronic moments. With a LF (filled circles) the interaction between the muon and local fields due to nuclear moments is quenched and this paramagnetic process is the only contribution to relaxation. For both materials studied a LF of $10 \mathrm{mT}$ was found to be sufficient to quench the nuclear moments, and further increases did not alter the decay of the muon polarization, even at the lowest temperature studied. The time constant for paramagnetic relaxation $\lambda$ was obtained by a straightforward fit of the LF $\mu$ SR signal to an exponential function. Its temperature variation is shown in Figure 4 for samples 2 with $x=0.43$ (red circles) and 3 with $x=0.58$ (blue squares) and qualitatively follows a Curie-Weiss law as expected from magnetic susceptibility measurements (Table 3). The depolarization rate and the muon hopping rate were extracted at each temperature from the ZF $\mu \mathrm{SR}$ signal by fitting to a dynamic KuboToyabe function damped by a fixed $\lambda$ taken from the corresponding LF experiment. The baseline asymmetry was used as a third fitting parameter.

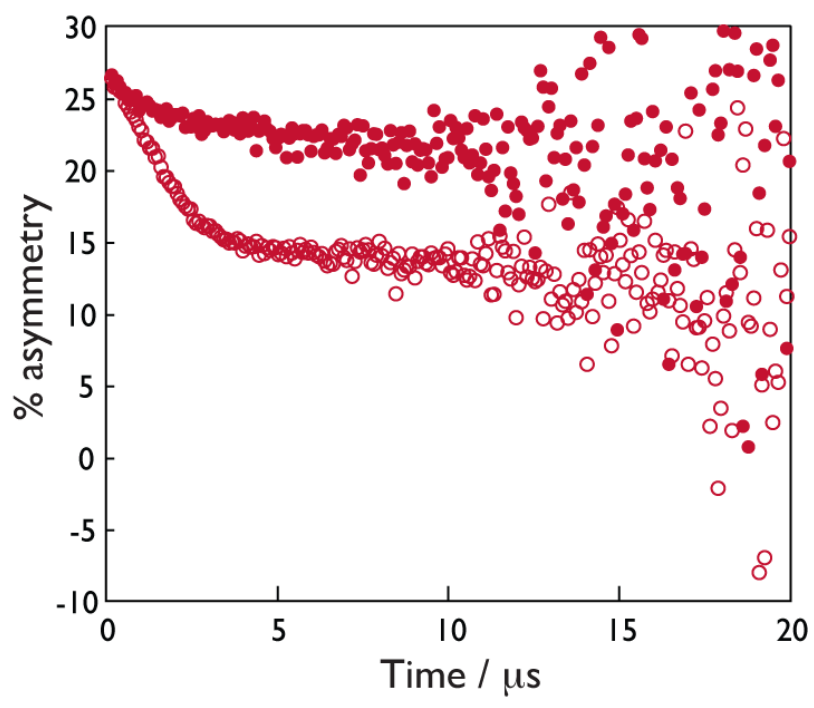

Figure 3. (Colour) Typical ZF $\mu \mathrm{SR}$ signal (open circles) obtained from $\mathrm{Li}_{3-x-y} \mathrm{Co}_{x} \mathrm{~N}$ materials, in this case sample 3 with $x=0.58$ recorded at $20 \mathrm{~K}$. Also LF $\mu$ SR signals (filled circles) recorded with a longitudinal magnetic field of $10 \mathrm{mT}$. Error bars increase with time due to the radioactive decay of the muons and have been omitted for clarity. 


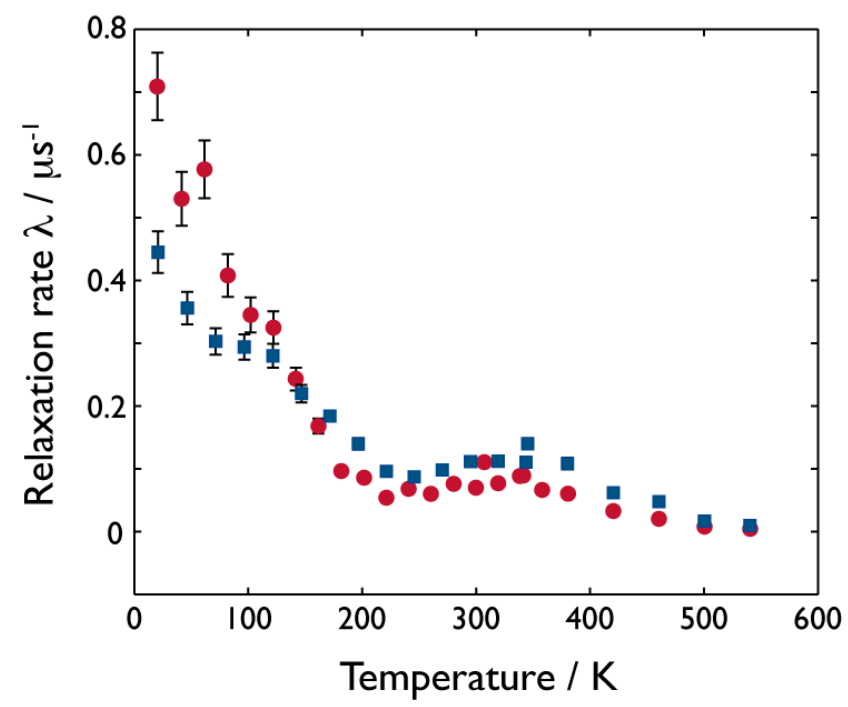

Figure 4. (Colour) Relaxation rate $\lambda$ extracted from variable-temperature LF $\mu$ SR measurements carried out as described in the text for $\mathrm{Li}_{3-\mathrm{x}-\mathrm{y}} \mathrm{Co}_{\mathrm{x}} \mathrm{N}$ with $\mathrm{x}=0.43(\mathbf{2}$, red circles) and $0.58(\mathbf{3}$, blue squares). Error bars are within the symbols at temperatures above $150 \mathrm{~K}$.

Values of $\Delta$ extracted from the $\mu \mathrm{SR}$ data in this way are plotted in Figure 5 for 2 with $\mathrm{x}=0.43$ (red circles) and 3 with $x=0.58$ (blue squares) for temperatures above $66 \mathrm{~K}$. The depolarization rate is approximately constant for both materials below $190 \mathrm{~K}$ with $\Delta=0.53 \pm 0.02 \mu \mathrm{s}^{-1}$ and $0.54 \pm 0.02 \mu \mathrm{s}^{-1}$, respectively. The lowtemperature values for $\mathrm{Li}_{3-x-y} \mathrm{Co}_{x} \mathrm{~N}$ are significantly higher than those for Ni-substituted materials, ${ }^{4}$ as expected because of the replacement of ${ }^{58,60} \mathrm{Ni}$ with $\mathrm{I}=0$ by ${ }^{59} \mathrm{Co}$ with $\mathrm{I}=7 / 2$. Above $200 \mathrm{~K}$ there is a rapid fall to less than $20 \%$ of the low-temperature $\Delta$. The muon hopping rate $v$ obtained from the dynamic KuboToyabe fits is plotted for temperatures above $190 \mathrm{~K}$ in Figure 6 for 2 with $\mathrm{x}=0.43$ (red circles) and 3 with $\mathrm{x}=$ 0.58 . The muon hopping rate does not vary significantly with temperature in this range with $v=0.46 \pm 0.02$ and $0.51 \pm 0.02 \mu$ s for 2 and 3, respectively. This implies that above $190 \mathrm{~K}$ the muon is quasi-static with a temperature-independent mean residence time $1 / v$ longer than its half-life. 


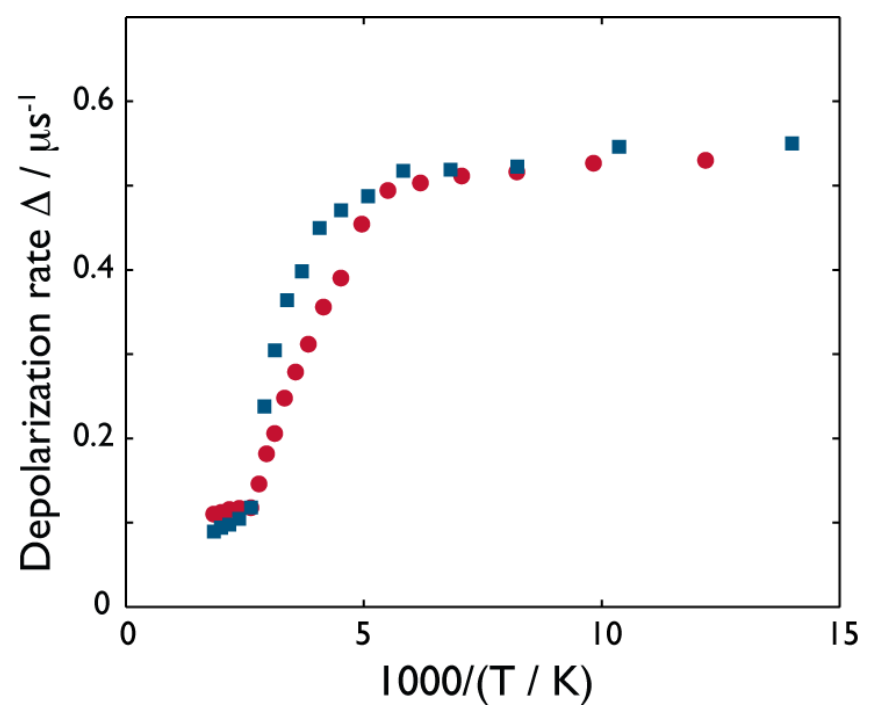

Figure 5. (Colour) Kubo-Toyabe depolarization rate for $\mathrm{Li}_{3-x-y} \mathrm{Co}_{x} \mathrm{~N}$ materials with $\mathrm{x}=0.43$ (2, red circles) and 0.58 (3, blue squares). Note that the error on $\Delta$ ranges from \pm 0.005 to \pm 0.014 and that the error bars are therefore inside the symbols for all temperatures.

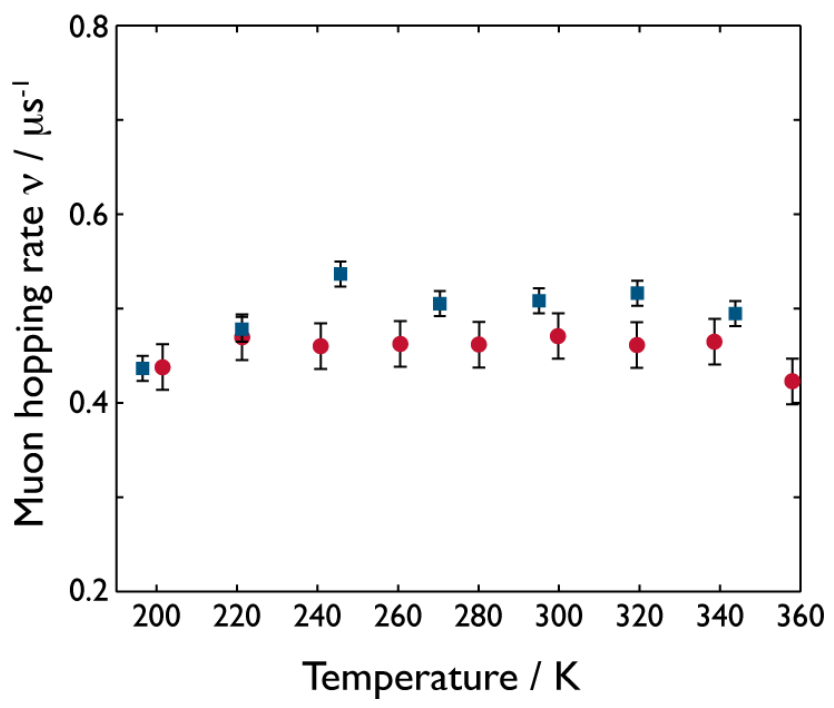

Figure 6. (Colour) Muon hopping rates obtained from fit to the dynamic Kubo-Toyabe function for $\mathrm{Li}_{3-\mathrm{x}-}$ ${ }_{y} \mathrm{Co}_{x} \mathrm{~N}$ materials with $\mathrm{x}=0.43(2$, red circles) and 0.58 (3, blue squares).

Muon Implantation Sites. The depolarization rate can be calculated for a powder sample by summing over the dipolar interactions between the muon and each nuclear spin in the surrounding crystal lattice. Table 4 shows values of $\Delta$ for several possible muon sites calculated using the lattice parameters obtained from neutron diffraction following the procedure described by Powell et al. ${ }^{12}$ (see the Supplementary Information for further details). Plausible choices for muon implantation include the Wyckoff $2 c$ site which corresponds to a $\operatorname{Li}(2)$ vacancy in the $\left[\mathrm{Li}_{2} \mathrm{~N}\right] a b$ plane and the $2 d$ interstitial located directly above the $2 c$ site in the 
$\operatorname{Li}(1) /$ Co plane which is the most open site in the structure. However, the experimental values of $\Delta$ for both Co-substituted materials are substantially larger than those calculated in Table 4 for either of these sites. Significantly better agreement with experiment is obtained if the muon is displaced along the crystallographic $c$ axis away from the $2 d$ interstitial towards a $2 c \operatorname{Li}(2)$ site, and muon implantation in this Wyckoff $4 h$ site with a displacement equal to $10 \%$ of the $c$ lattice parameter was suggested previously for $\mathrm{Li}_{3-}$ ${ }_{x-y} \mathrm{Ni}_{x} \mathrm{~N} .{ }^{4}$ Interestingly, a similar interstitial location has been identified by molecular dynamics simulations ${ }^{25}$ as an intermediate $\mathrm{Li}^{+}$site in the exchange between $\mathrm{Li}(1)$ and $\mathrm{Li}(2)$ required for inter-layer diffusion in the parent $\mathrm{Li}_{3} \mathrm{~N}$. As the degree of Co substitution increases, the calculated $\Delta$ for all sites decreases slightly, as expected, because of the replacement of ${ }^{7} \mathrm{Li}$ magnetic moments by ${ }^{59} \mathrm{Co}$ and the expansion of the $\left[\mathrm{Li}_{2} \mathrm{~N}\right] a b$ plane. However, the errors on the experimental values of $\Delta$ are such that these small variations cannot be detected. Other possibilities for muon sites, such as in a $\operatorname{Li}(2)$ vacancy adjacent to a possible $\mathrm{NH}^{2}$ - defect or close to the $\mathrm{N}^{3-}$ ion in the $\left[\mathrm{Li}_{2} \mathrm{~N}\right]$ plane, result in calculated $\Delta$ values which are much higher than those observed experimentally.

Table 4 also shows depolarization rates calculated with either the $\operatorname{Li}(2)$ atoms only or all the $\mathrm{Li}$ atoms removed from the structure. The former represent the residual contribution to the muon depolarization in the case that the intra-layer $\mathrm{Li}^{+}$jump rate substantially exceeds the low-temperature limiting value of $\Delta$. The latter also take account of similarly rapid inter-layer $\mathrm{Li}^{+}$diffusion and are significantly higher than their counterparts in $\mathrm{Li}_{3-\mathrm{x}-\mathrm{y}} \mathrm{Ni}_{\mathrm{x}} \mathrm{N}$ materials with similar $\mathrm{x} .{ }^{4}$ Once again, this is expected given the replacement of ${ }^{58,60} \mathrm{Ni}$ with $\mathrm{I}=0$ by ${ }^{59} \mathrm{Co}$ with $\mathrm{I}=7 / 2$. The calculated depolarization rates suggest that for Co-substituted materials $\mu \mathrm{SR}$ is particularly sensitive to intra-layer diffusion, since $\Delta$ decreases to approximately half its low-temperature limit with rapid $\mathrm{Li}^{+}$jumps between $\mathrm{Li}(2)$ sites. 


\begin{tabular}{||l|c|c|c|c||}
\hline \hline Site & $\begin{array}{c}\text { Wyckoff } \\
\text { notation }\end{array}$ & Co-ordinates & $\begin{array}{c}\mathbf{2} \\
\mathrm{x}=0.43\end{array}$ & $\begin{array}{c}\mathbf{3} \\
\mathrm{x}=0.58\end{array}$ \\
\hline \hline All atoms present & & & & \\
\hline $\mathrm{Li}(2)$ vacancy in $[\mathrm{Li} 2 \mathrm{~N}] a b$ plane & $2 c$ & $(1 / 3,2 / 3,0)$ & 0.24 & 0.22 \\
\hline Interstitial site in $\mathrm{Li}(1) / \mathrm{Ni}$ plane & $2 d$ & $(1 / 3,2 / 3,1 / 2)$ & 0.45 & 0.44 \\
\hline Interstitial with z displacement & $4 h$ & $(1 / 3,2 / 3, \mathrm{z})$ with $\mathrm{z}=2 / 5$ & 0.52 & 0.51 \\
\hline \hline $\mathrm{Li}(\mathbf{2})$ removed & & & \\
\hline $\mathrm{Li}(2)$ vacancy in $[\mathrm{Li} 2 \mathrm{~N}] a b$ plane & $2 c$ & $(1 / 3,2 / 3,0)$ & 0.19 & 0.20 \\
\hline Interstitial site $\mathrm{Li}(1) / \mathrm{Ni}$ plane & $2 d$ & $(1 / 3,2 / 3,1 / 2)$ & 0.29 & 0.30 \\
\hline Interstitial with z displacement & $4 h$ & $(1 / 3,2 / 3, \mathrm{z})$ with $\mathrm{z}=2 / 5$ & 0.28 & 0.29 \\
\hline \hline $\mathrm{Li}(\mathbf{1})$ and $\mathrm{Li}(\mathbf{2})$ removed & & & & \\
\hline $\mathrm{Li}(2)$ vacancy in $\left[\mathrm{Li}{ }_{2} \mathrm{~N}\right] a b$ plane & $2 c$ & $(1 / 3,2 / 3,0)$ & 0.14 & 0.17 \\
\hline Interstitial site $\mathrm{Li}(1) / \mathrm{Ni}$ plane & $2 d$ & $(1 / 3,2 / 3,1 / 2)$ & 0.22 & 0.25 \\
\hline Interstitial with z displacement & $4 h$ & $(1 / 3,2 / 3, \mathrm{z})$ with $\mathrm{z}=2 / 5$ & 0.21 & 0.24 \\
\hline
\end{tabular}

Table 4. Calculated values of the Kubo-Toyabe depolarization rate $\Delta$ in $\mu s^{-1}$ for $\operatorname{Li}_{3-x-y} \mathrm{Co}_{x} N$ materials with $x=$ $0.43(2)$ and $0.58(3)$.

a. corresponds to the residual contribution to depolarization rate when the intra-layer $\mathrm{Li}^{+}$jump rate substantially exceeds the low-temperature limiting value of $\Delta$.

b. corresponds to the residual contribution to depolarization rate when both intra- and inter-layer $\mathrm{Li}^{+}$jump rates substantially exceeds the low-temperature limiting value of $\Delta$.

Lithium Diffusion in $\mathrm{Li}_{3-x-y} C o_{x} N$. The significant decrease in the depolarization rate observed for both samples at temperatures above $190 \mathrm{~K}$ does not originate from muon dynamics, since the muon is quasi-static in this regime, as confirmed by the temperature-independent hopping rate. Instead, the reduction in $\Delta$ originates from motional narrowing of a contribution to the local field distribution at the muon due to the onset of $\mathrm{Li}^{+}$ diffusion within the $\left[\mathrm{Li}_{2} \mathrm{~N}\right]$ plane. At $300 \mathrm{~K}$ the experimental depolarization rate (Figure 5) is approximately equal to the value calculated (Table 4) with the $\operatorname{Li}(2)$ atoms removed from the structure, suggesting that the intra-layer $\mathrm{Li}^{+}$jump rate substantially exceeds the low-temperature limiting value of $\Delta$ by this temperature. Similar motional narrowing for a quasi-static muon in ZF $\mu$ SR has been attributed previously to $\mathrm{Li}^{+}$diffusion in lithium manganate spinels ${ }^{2}$, as well as for the parent $\mathrm{Li}_{3} \mathrm{~N}$ and $\mathrm{Li}_{3-\mathrm{x}-\mathrm{y}} \mathrm{Ni}_{\mathbf{x}} \mathrm{N} .{ }^{4}$ In contrast, Sugiyama et al. ${ }^{3}$ found an activated $v$, coupled with decrease in $\Delta$ for a muon forming a hydrogen-like bond to oxygen in $\mathrm{Li}_{x} \mathrm{CoO}_{2}$. Only the contribution which arises from dipolar interactions with ${ }^{6} \mathrm{Li}$ or ${ }^{7} \mathrm{Li}$ nuclei is narrowed by $\mathrm{Li}^{+}$diffusion. The line narrowing has been modelled in this work by ${ }^{26}$

$$
\Delta^{2}=\Delta_{0}^{2} \frac{2}{\pi} \tan ^{-1}\left(\Delta \pi \tau_{c}\right)
$$


where $\tau_{\mathrm{c}}$ is the mean residence time for diffusing $\mathrm{Li}^{+}$ions and $\Delta_{0}$ is the low-temperature limiting value of $\Delta$. For intra-layer diffusion $\Delta_{0}$ was taken as the average of all measurements below $190 \mathrm{~K}$. The $\mathrm{Li}^{+}$jump rates $1 / \tau_{\mathrm{c}}$ extracted from Eq. (2) in this way are in the $\mathrm{MHz}$ regime at room temperature, and the assumption of a simple Arrhenius law allows an activation energy to be obtained from the temperature variation. Arrhenius plots shown in Figure 7 for 2 (open red circles) and 3 (open blue squares) indicate that this model provides a good fit to the data for temperatures from the onset of motional narrowing at $190 \mathrm{~K}$ up to about $300 \mathrm{~K}$. The resulting intra-layer $E_{a}$ and intrinsic jump rates $1 / \tau_{c, 0}\left(1 / \tau_{c}\right.$ at $\left.T=\infty\right)$ are given in Table 5 .

Above $300 \mathrm{~K}$ the depolarization rate continues to decrease beyond the value calculated for rapid intra-layer $\mathrm{Li}^{+}$diffusion. This implies the onset of inter-layer $\mathrm{Li}^{+}$jumps at a rate sufficient to motionally narrow the residual contribution to $\Delta$ arising from dipolar interactions between the muon and $\mathrm{Li}$ nuclei on $\mathrm{Li}(1)$ sites. Jump rates for inter-layer diffusion were obtained by setting $\Delta_{0}$ equal to the value calculated with the $\mathrm{Li}(2)$ atoms removed from the structure (Table 4). The corresponding Arrhenius plots for 2 (filled red circles) and 3 (filled blue squares) are also shown in Figure 7, and the resulting inter-layer activation energies and intrinsic jump rates are given in Table 5. The narrow temperature range over which $\Delta$ is sensitive to interlayer diffusion and the lack of an experimental value for $\Delta_{0}$ these parameters prevent a more accurate determination. For $1 / \tau_{\mathrm{c}, 0}$ the errors are so significant that only an order of magnitude is given in Table 5 , but this is around $10^{13} \mathrm{~Hz}$ as expected for a lattice vibration. Note that similar high-temperature inter-layer contributions to diffusion were not measured in $\mu \mathrm{SR}$ studies of the Ni-substituted materials ${ }^{4}$, since the maximum temperature in those experiments was only $350 \mathrm{~K}$.

Trends in Diffusion Parameters for $L i_{3-x-y} M_{x} N$. For intra-layer diffusion the effect of metal substitution on the activation energy revealed by $\mu S R$ and NMR studies can be rationalized in terms of the structural information obtained from neutron diffraction. As $x$ increases the lattice parameter $a$ in $\mathrm{Li}_{3-\mathrm{x}-\mathrm{y}} \mathrm{Co}_{\mathrm{x}} \mathrm{N}$ increases and the $\mathrm{Li}-\mathrm{N}$ distance within the $\left[\mathrm{Li}_{2} \mathrm{~N}\right]$ planes lengthens, resulting in a more open pathway for $\mathrm{Li}^{+}$ diffusion through the expanded layers and a decrease in the intra-layer $E_{a}$. However, the accompanying decrease in the lattice parameter $c$ corresponds to an increase in the covalent character of the Li - $\mathrm{N}$ bond which in turn reduces the polarizability of the lattice framework and increases the energy barrier. For the two $\mathrm{Li}_{3-x-y} \mathrm{Co}_{x} \mathrm{~N}$ materials the activation energy for intra-layer diffusion obtained with $\mu \mathrm{SR}$ increases as the degree of substitution increases, suggesting that the most important effect in this case is the reduction in the $c$ lattice parameter. This situation is similar to that observed for two Ni-substituted materials ${ }^{4,10}$ with $x>0.36$, 
but in contrast to that observed for three members of the $\mathrm{Li}_{3-\mathrm{x}-\mathrm{y}} \mathrm{Cu}_{\mathrm{x}} \mathrm{N}$ series with $\mathrm{x}<0.35 .12$ Figure 8 shows the variation in the inter-layer $\mathrm{E}_{\mathrm{a}}$ with the lattice parameters $a$ and $c$ for $\mathrm{Li}_{3-\mathrm{x}-\mathrm{y}} \mathrm{M}_{\mathrm{x}} \mathrm{N}$ with $\mathrm{M}=\mathrm{Cu}, \mathrm{Ni}, \mathrm{Co}$. The plot suggests that an optimal substitution level exists which minimizes the energy barrier for $\mathrm{Li}^{+}$hopping. This optimum corresponds to a common value for the $a$ parameter of around $3.69 \AA$, regardless of M. For this value the beneficial effect of expanding the $\mathrm{Li}-\mathrm{N}$ distance within the $\left[\mathrm{Li}_{2} \mathrm{~N}\right]$ layers still dominates, but any further expansion in $a$ is accompanied by a detrimental contraction in $c$. In contrast to substitution level, vacancy concentration has little effect on intra-layer diffusion. The $\mathrm{Li}_{3-x-y} \mathrm{Co}_{x} \mathrm{~N}$ materials measured here show slightly lower intrinsic jump rates than their $\mathrm{Cu}$-substituted counterparts, ${ }^{12}$ despite the vanishing vacancy concentrations of the latter. Note, however, that this is not unexpected in the light of the complex mechanisms for $\mathrm{Li}^{+}$transport in the parent $\mathrm{Li}_{3} \mathrm{~N}$ suggested by molecular dynamics calculations. ${ }^{25}$ Interestingly, while magnetic measurements indicate significant differences in bonding between the layers for different substituent metals, intra-layer diffusion remains essentially unaffected. For inter-layer diffusion $\mathrm{E}_{\mathrm{a}}$ for $\mathrm{Li}_{3-\mathrm{x}-\mathrm{y}} \mathrm{Co}_{\mathrm{x}} \mathrm{N}$ with $\mathrm{x}=0.43$ is comparable to that obtained previously12 for the members of the $\mathrm{Li}_{3-\mathrm{x}-\mathrm{y}} \mathrm{Cu}_{\mathrm{x}} \mathrm{N}$ series. However, there is a significant rise in $E_{a}$ as the substitution level increases to $x=0.58$ which may reflect the increased electronic delocalization associated with inter-layer bonding as revealed by magnetic measurements.

\begin{tabular}{||c|c|c||}
\hline Sample, composition & $\mathbf{2}, \mathrm{x}=0.43$ & $\mathbf{3}, \mathrm{x}=0.58$ \\
\hline \hline \multicolumn{3}{|c||}{ Intra-layer Diffusion } \\
\hline \hline $\mathrm{E}_{\mathrm{a}} / \mathrm{eV}$ & $0.072 \pm 0.004$ & $0.081 \pm 0.008$ \\
\hline$\left(1 / \boldsymbol{\tau}_{\mathrm{c}, 0}\right) / 10^{7} \mathrm{~Hz}$ & $4.4 \pm 0.9$ & $3.6 \pm 1.4$ \\
\hline \multicolumn{3}{|c||}{ Inter-layer Diffusion } \\
\hline \hline $\mathrm{E}_{\mathrm{a}} / \mathrm{eV}$ & $0.40 \pm 0.1$ & $0.57 \pm 0.06$ \\
\hline$\left(1 / \tau_{\mathrm{c}, 0}\right) / \mathrm{Hz}$ & $10^{12}$ & $10^{13}$ \\
\hline
\end{tabular}

Table 5. Activation energies and intrinsic jump rates extracted from ZF $\mu$ SR measurements for $\mathrm{Li}_{3-\mathrm{x}-\mathrm{y}} \mathrm{Co}_{\mathrm{x}} \mathrm{N}$ materials with $x=0.43(2)$ and $0.58(3)$. 


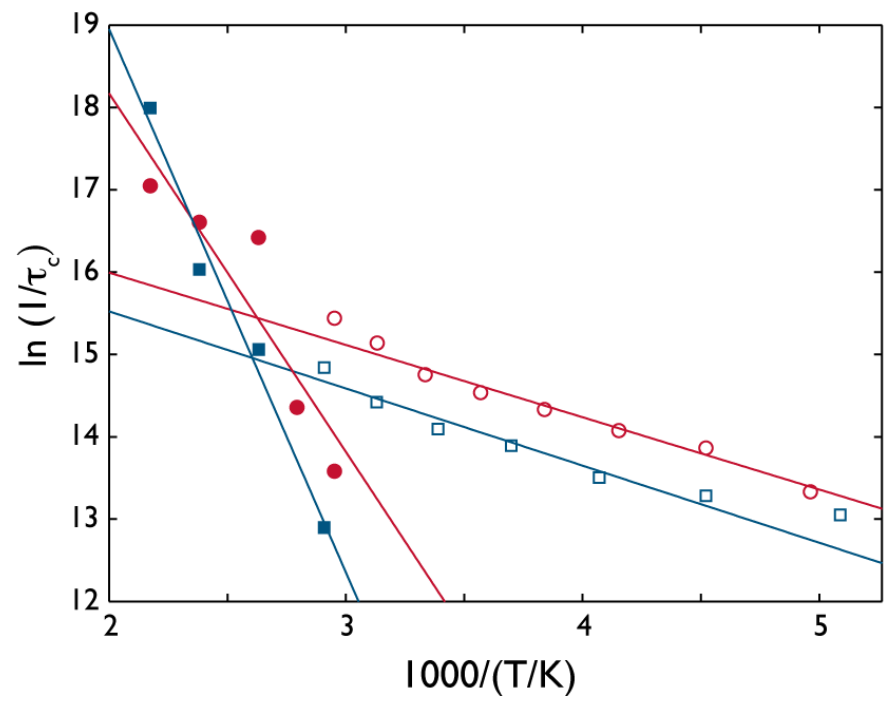

Figure 7. (Colour) Arrhenius plots of $\mathrm{Li}^{+}$jump rates $1 / \tau_{\mathrm{c}}$ measured from $\mathrm{ZF} \mu \mathrm{SR}$ experiments for $\mathrm{Li}_{3-\mathrm{x}-\mathrm{y}} \mathrm{Co}_{\mathrm{x}} \mathrm{N}$ materials with $x=0.43(2$, red circles) and $x=0.57$ (3, blue squares) for intra-layer (open symbols) and interlayer diffusion (filled symbols), together with fits (lines) used to extract activation energies.
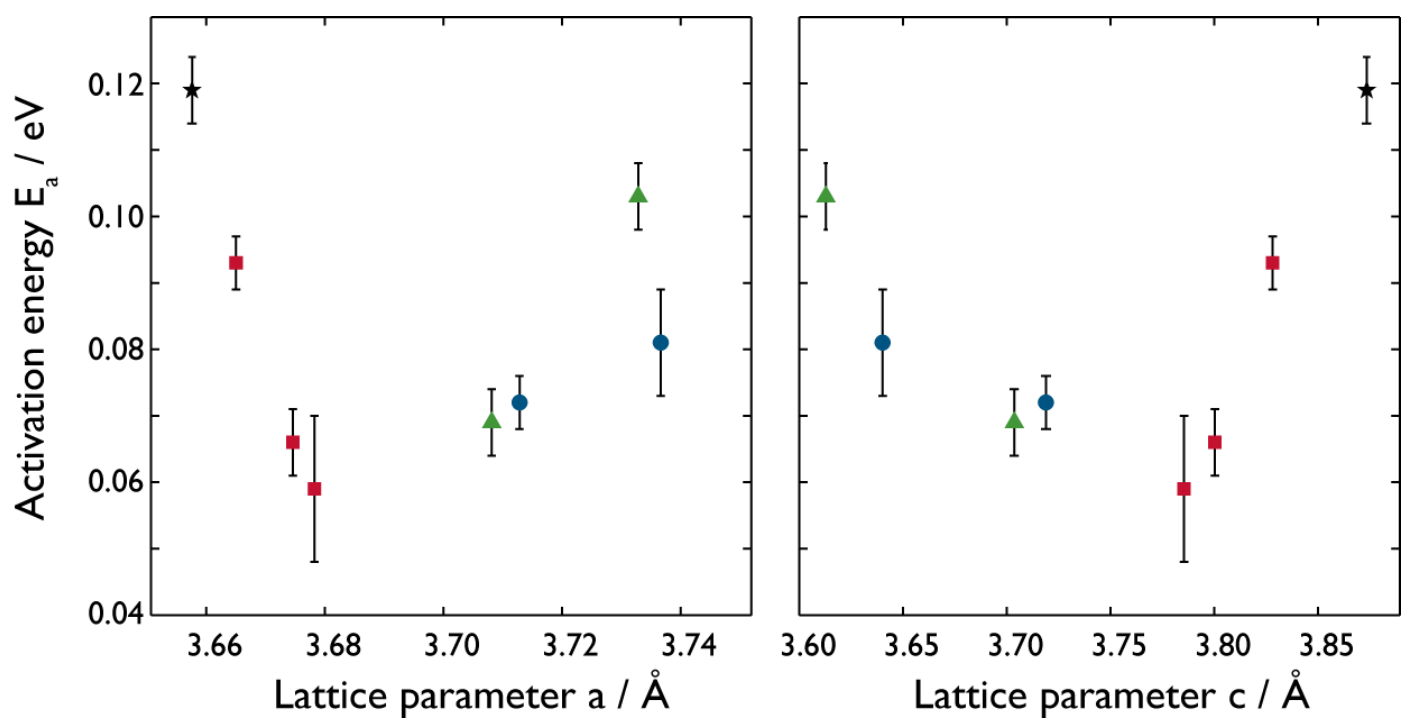

Figure 8. (Colour) Activation energy for intra-layer diffusion in $\mathrm{Li}_{3-\mathrm{x}-\mathrm{y}} \mathrm{M}_{\mathrm{x}} \mathrm{N}$ for $\mathrm{M}=\mathrm{Co}$ (blue circles, this work), $\mathrm{Ni}$ (green triangles) ${ }^{4}$ and $\mathrm{Cu}$ (red squares) ${ }^{12}$ as a function of lattice parameters $a$ (top) and $c$ (bottom), as well as for $\mathrm{Li}_{3} \mathrm{~N}$ (black star).

\section{Conclusion}

PND shows that the three lithium cobalt nitride materials studied here retain the $P 6 / \mathrm{mmm}$ space group of $\mathrm{Li}_{3} \mathrm{~N}$ with Co located only on $\operatorname{Li}(1)$ sites. The lattice parameters $a$ and $c$ vary smoothly with $\mathrm{x}$ in a similar fashion to similar series of $\mathrm{Cu}$ - and Ni-substituted materials.12,13 In contrast to the latter, the $\mathrm{Li}_{3-x-y} \mathrm{Co}_{x} \mathrm{~N}$ phases exhibit Curie-Weiss paramagnetism which precludes the use of NMR to gain atomic-level insight 
into $\mathrm{Li}^{+}$transport. However, the results described here demonstrate that $\mu \mathrm{SR}$ can be employed as an alternative technique to study these paramagnetic materials, and that quantitative information about $\mathrm{Li}^{+}$ diffusion can be obtained. Insight gained from $\mu \mathrm{SR}$ measurements shows that $\mathrm{Li}^{+}$diffusion is anisotropic in a similar fashion to that detected in $\mathrm{Li}_{3} \mathrm{~N}$ and in $\mathrm{Li}_{3-x} \mathrm{Cu}_{x} \mathrm{~N}$ by $\mathrm{NMR}^{12,26}$ with transport confined to the [ $\left[\mathrm{Li}_{2} \mathrm{~N}\right.$ ] plane at low temperature and exchange between $\operatorname{Li}(1)$ and $\operatorname{Li}(2)$ sites dominant at high temperature. In conjunction with previous NMR, $\mu \mathrm{SR}$ and PND studies ${ }^{4,12,13}$ the new data for $\mathrm{Li}_{3-x-y} \mathrm{Co}_{x} \mathrm{~N}$ allow some general trends to be established across a range of $\mathrm{Li}_{3-x-y} \mathrm{M}_{x} \mathrm{~N}$ materials. For intra-layer diffusion $\mathrm{E}_{\mathrm{a}}$ initially decreases with $\mathrm{x}$ as the corresponding expansion in $a$ results in a more open pathway for $\mathrm{Li}^{+}$diffusion through the layers. However, an optimal value of $\mathrm{x}$ is reached with $a \approx 3.69 \AA$ after which the concomitant contraction in c reduces the polarizability of the lattice framework. By contrast, considering all the $\operatorname{Li}_{3-x-y} M_{x} N$ materials together, there appears to be no correlation between intra-layer diffusion and vacancy concentration.

\section{Acknowledgements}

This research was supported in its initial stages by EPSRC grant GR/R87345. Experiments at the ISIS Pulsed Neutron and Muon Source were made possible by beamtime allocations from the STFC. ASP thanks the EPSRC and the University of Nottingham for a PhD studentship.

\section{References}

(1) Gee, B.; Horne, C.; Cairns, E.; Reimer, J. J. Phys. Chem. B 1998, 102, 10142-10149.

(2) Kaiser, C.; Verhoeven, V.; Gubbens, P.; Mulder, F.; de Schepper, I.; Yaouanc, A.; de Reotier, P.; Cottrell, S.; Kelder, E.; Schoonman, J. Phys. Rev. B 2000, 62, R9236-R9239.

(3) Sugiyama, J.; Mukai, K.; Ikedo, Y.; Nozaki, H.; Månsson, M.; Watanabe, I. Phys. Rev. Lett. 2009, $103,147601$.

(4) Powell, A. S.; Lord, J. S.; Gregory, D. H.; Titman, J. J. J. Phys. Chem. C 2009, 113, 20758-20763.

(5) Hayano, R.; Uemura, Y.; Imazato, J.; Nishida, N.; Yamazaki, T.; Kubo, R. Phys. Rev. B 1979, $20,850-859$.

(6) Shodai, T.; Okada, S.; Tobishima, S.; Yamaki, J. Solid State Ionics 1996, 86-8, 785-789.

(7) Shodai, T.; Okada, S.; Tobishima, S.; Yamaki, J. J. Power Sources 1997, 68, 515-518.

(8) Gregory, D. H.; O'Meara, P. M.; Gordon, A. G.; Siddons, D. J.; Blake, A. J.; Barker, M. G.; Hamor, T. A.; Edwards, P. P. J. Alloy Cmpd. 2001, 317, 237-244.

(9) Gordon, A. G.; Gregory, D. H.; Blake, A. J.; Weston, D. P.; Jones, M. O. Int. J. Inorg. Mater. 2001, 3, $973-981$.

(10) Gregory, D. H.; O'Meara, P. M.; Gordon, A. G.; Hodges, J. P.; Short, S.; Jorgensen, J. D. Chem. Mater. 2002, 14, 2063-2070.

(11) Schulz, H.; Thiemann, K. H. Acta Crystallogr. A 1979, 35, 309-314. 
(12) Powell, A. S.; Stoeva, Z.; Smith, R. I.; Gregory, D. H.; Titman, J. J. Phys. Chem. Chem. Phys. 2011, 13, 10641-10647.

(13) Stoeva, Z.; Smith, R. I.; Gregory, D. H. Chem. Mater. 2006, 18, 313-320.

(14) Barker, M. G.; Begley, M. J.; Edwards, P. P.; Gregory, D. H.; Smith, S. E. J. Chem. Soc. Dalton Trans. 1996, 1-5.

(15) Kraus, W.; Nolze, G. J. Appl. Crystallogr. 1996, 29, 301-303.

(16) Larson, A. C.; von Dreele, R. B. The General Structure Analysis System, Report No. LAUR 086-748, Los Alamos National Laboratories: Los Alamos, 2000.

(17) Toby, B. H. J. Appl. Crystallogr. 2001, 34, 210-213.

(18) Boultif, A.; Louer, D. J. Appl. Crystallogr. 1991, 24, 987-993.

(19) Pratt, F. L. Physica B, 2000, 289, 710-714.

(20) Sachsze, W.; Juza, R. Z. Anorg. Allg. Chem. 1949, 259, 278.

(21) Nishijima, M.; Kagohashi, T.; Imanishi, M.; Takeda, Y.; Yamamoto, O.; Kondo, S. Solid State Ionics, 1996, 83, 107111.

(22) Niewa, R.; Huang, Z.-L.; Schnelle, W.; Hu, Z.; Kniep, R. Z. Anorg. Allg. Chem. 2003, 629, 1778.

(23) Schnelle, W. ; Niewa, R.; Wagner, F. R. J. Magn. Magn. Mater., 2004, 272-276, 828-829.

(24) Suzuki, S.; Shodai, T.; Yamaki, J. J. Phys. Chem. Solids, 1998, 59, 331-336.

(25) Wolf, M. L.; Walker, J. R.; Catlow, C. J. Phys. C Solid State 1984, 17, 6623-6634.

(26) Messer, R.; Birli, H.; Differt, K. J. Phys. C Solid State 1981, 14, 2731-2746. 